\title{
Łojasiewicz exponents and resolution of singularities
}

\author{
C. Bivià-Ausina And S. Encinas
}

\begin{abstract}
We show an effective method to compute the Łojasiewicz exponent of an arbitrary sheaf of ideals of $\mathcal{O}_{X}$, where $X$ is a non-singular scheme. This method is based on the algorithm of resolution of singularities.
\end{abstract}

Mathematics Subject Classification (2000). Primary 2S05, 14E15;

Secondary $13 \mathrm{H} 15$.

Keywords. Resolution of singularities, Łojasiewicz exponents.

1. Introduction. Given an analytic function $f:\left(\mathbb{C}^{n}, 0\right) \rightarrow(\mathbb{C}, 0)$ with an isolated singularity at the origin, the effective computation of the Lojasiewicz exponent $\mathcal{L}_{0}(f)$ of $f$ is a problem that has been approached from both algebraic and analytic techniques (see for instance $[1,6,13,15]$ ). This number is defined as the infimum of those real numbers $\alpha>0$ such that

$$
\|x\|^{\alpha} \leqslant C\|\nabla f(x)\|
$$

for some constant $C>0$ and all $x$ belonging to some open neighbourhood of the origin in $\mathbb{C}^{n}$, where $\nabla f$ denotes the gradient of $f$. One of the most significant applications of $\mathcal{L}_{0}(f)$ is the result of Teissier [17, p. 280] stating that the degree of topological determinacy of $f$ is equal to $\left[\mathcal{L}_{0}(f)\right]+1$, where $[a]$ denotes the integer part of a number $a \in \mathbb{R}$. Let us denote by $j^{r} f$ the $r$-jet of $f$, that is, the sum of all terms of the Taylor expansion of $f$ around the origin of degree $\leqslant r$. Then the degree of topological determinacy of $f$ is defined as the minimum of those $r \geqslant 1$ such that for all $g \in \mathcal{O}_{n}$ verifying that $j^{r} f=j^{r} g$, we have that $f$ and $g$ are topologically equivalent, that is, there exists a germ of homeomorphism $\varphi:\left(\mathbb{C}^{n}, 0\right) \rightarrow\left(\mathbb{C}^{n}, 0\right)$ such that $f=g \circ \varphi$.

Let us denote by $\mathcal{O}_{n}$ the ring of analytic functions $f:\left(\mathbb{C}^{n}, 0\right) \rightarrow \mathbb{C}$. The definition of Łojasiewicz exponent of functions with an isolated singularity is

The first author was partially supported by DGICYT Grant MTM2006-06027. The second author was partially supported by DGICYT Grant MTM2006-10548. 
extended naturally to ideals of $\mathcal{O}_{n}$ of finite colength. Let $I$ be an ideal of $\mathcal{O}_{n}$. In this article we apply the explicit construction of a log-resolution of $I$ given in [2] to compute effectively the Eojasiewicz exponent $\mathcal{L}_{0}(I)$ of $I$ provided that $I$ has finite colength. We consider the problem of computing $\mathcal{L}_{0}(I)$ in a more general setting, that is, we substitute $I$ by a sheaf of ideals in a non-singular scheme.

As an application of the main result, we compute the Łojasiewicz exponent, and consequently the degree of topological determinacy, of a function such that $\mathcal{L}_{0}(f)$ can not be computed by means of the existing literature about this subject.

2. Order functions. In this section we recall some known facts concerning the integral closure of ideals and its relation with reduced orders. We will denote by $R$ a Noetherian ring.

Definition 2.1. Let $\overline{\mathbb{R}}_{0}=\{a \in \mathbb{R} \mid a \geqslant 0\} \cup\{\infty\}$ and let us consider a function $\rho: R \rightarrow \overline{\mathbb{R}}_{0}$. We say that $\rho$ is an order function if the following conditions hold:

(i) $\rho(f+g) \geqslant \min \{\rho(f), \rho(g)\}$, for all $f, g \in R$.

(ii) $\rho(f g) \geqslant \rho(f)+\rho(g)$, for all $f, g \in R$.

(iii) $\rho(0)=\infty$ and $\rho(1)=0$.

Let $I \subseteq R$ be an ideal and let $f \in R$. It is well known, and also easy to prove, that the function

$$
\nu_{I}(f)=\sup \left\{m \in \mathbb{N} \mid f \in I^{m}\right\}
$$

is an order function. Let $J \subseteq R$ be an ideal and set

$$
\nu_{I}(J)=\sup \left\{m \in \mathbb{N} \mid J \subseteq I^{m}\right\} .
$$

If $f_{1}, \ldots, f_{s}$ are generators of $J$, then it can be checked that

$$
\nu_{I}(J)=\min \left\{\nu_{I}\left(f_{1}\right), \ldots, \nu_{I}\left(f_{s}\right)\right\} .
$$

Proposition 2.2. [16][14, Section 0.2] Let $I \subseteq R$ be an ideal with $I \neq R$. Then the sequence

$$
\left\{\frac{\nu_{I}\left(f^{n}\right)}{n}\right\}_{n=1}^{\infty}
$$

has a limit in $\overline{\mathbb{R}}_{0}$. Moreover the function $\bar{\nu}_{I}: R \rightarrow \overline{\mathbb{R}}_{0}$ defined by

$$
\bar{\nu}_{I}(f)=\lim _{n \rightarrow \infty} \frac{\nu_{I}\left(f^{n}\right)}{n}
$$

is an order function.

The number $\bar{\nu}_{I}(f)$ is called the reduced order of $f$ with respect to $I$. It is proved in [14] that $\bar{\nu}_{I}(f) \in \mathbb{Q}_{+} \cup\{\infty\}$, for all $f \in R$. We will show this result using the existence of embedded desingularization of schemes and logresolution of ideals. 
Remark 2.3. The sequence $\left\{u_{n}=\frac{\nu_{I}\left(f^{n}\right)}{n}\right\}_{n=1}^{\infty}$ is not an increasing sequence, in general. However, it is straightforward to see that, for any integer $i \geqslant 2$, the subsequence $\left\{u_{i^{n}}\right\}_{n=1}^{\infty}$ is increasing, so that

$$
\bar{\nu}_{I}(f)=\lim _{n \rightarrow \infty} \frac{\nu_{I}\left(f^{n}\right)}{n}=\sup \left\{\frac{\nu_{I}\left(f^{n}\right)}{n} \mid n \in \mathbb{N}\right\}
$$

and $n \bar{\nu}_{I}(f) \geqslant \nu_{I}\left(f^{n}\right)$ for all $n$. In particular $\bar{\nu}_{I}(f) \geqslant \nu_{I}(f)$, for all $f \in R$.

Lemma 2.4. [14, 0.2.9] Let $I$ and $J$ be ideals of $R$ and let $p, q$ be positive integers. Then

$$
\bar{\nu}_{I^{p}}\left(J^{q}\right)(x)=\frac{q}{p} \bar{\nu}_{I}(J)
$$

For an ideal $I$ of $R$ we will denote by $\bar{I}$ the integral closure of $I$.

Lemma 2.5. $[14,1.15][11$, p. 138] Let $R$ be a Noetherian ring and let $I, J$ be ideals of $R$. If $J \subseteq \bar{I}$, then $\bar{\nu}_{I}(J) \geqslant 1$.

Definition 2.6. Let $I \subseteq R$ be an ideal. We define the function $\mu_{I}: R \rightarrow \overline{\mathbb{R}}_{0}$ as

$$
\mu_{I}(f)=\sup \left\{\frac{p}{q} \in \mathbb{Q}_{+} \mid f^{q} \in \overline{I^{p}}\right\} .
$$

As a consequence of [11, Proposition 10.5.2] (see also [14, Section 4.2]) the set of rational numbers involved in Definition 2.6 does not depend on the representatives $p, q$ of the rational number $\frac{p}{q}$.

Let us consider the graded ring $R[T]$, with the usual graduation on $T$. Let $R[I T] \subseteq R[T]$ be the subring $R[I T]=\oplus_{n} I^{n} T^{n}$. Let $f \in R$, we have that $f \in \bar{I}$ if and only if the homogeneous element $f T \in R[T]$ is in the integral closure of the ring $R[I T]$ in $R[T]$. It is well known (see, for instance, [11, p. 95]) that this integral closure is

$$
\overline{R[I T]}=\bigoplus_{n} \overline{I^{n}} T^{n} \subseteq R[T]
$$

Lemma 2.7. If $f^{q} \in \bar{I}$ and $g^{q} \in \bar{I}$ then $(f+g)^{q} \in \bar{I}$.

Proof. By assumption $f^{q} T$ and $g^{q} T$ are integral over $R[I T]$. We observe that the ring extension $R[T] \subseteq R\left[T^{\frac{1}{q}}\right]$ is finite. Then $f T^{\frac{1}{q}}$ and $g T^{\frac{1}{q}}$ are integral over $R[I T] \subseteq R\left[T^{\frac{1}{q}}\right]$. Therefore $(f+g) T^{\frac{1}{q}}$ is integral over $R[I T]$. Thus $(f+g)^{q} T$ is integral over $R[I T]$ and we conclude that $(f+g)^{q} \in \bar{I}$.

Proposition 2.8. Let $I$ be an ideal of $R$. Then $\mu_{I}$ is an order function.

Proof. The fact that $\mu_{I}$ satisfies condition (i) of Definition 2.1 follows as a direct application of Lemma 2.7. Conditions (ii) and (iii) follow easily from the definition of $\mu_{I}$. 
3. Resolution of singularities and integral closure. In this section, $X$ will denote an integral separated scheme of finite type over a field $k$, where the characteristic of $k$ is zero.

If $\mathcal{I} \subseteq \mathcal{O}_{X}$ is a sheaf of ideals then the integral closure $\overline{\mathcal{I}}$ is a sheaf of ideals such that for every point $x \in X$, the ideal $\overline{\mathcal{I}}_{x}$ is the integral closure of $\mathcal{I}_{x} \subseteq \mathcal{O}_{X, x}$.

The next result is well known and its proof can be found, for instance, in [11, p. 133].

Lemma 3.1. Let $R$ be a Noetherian domain. Denote by $K$ the field of fractions of $R$. Let $I \subseteq R$ be an ideal. For every valuation ring $R_{v} \subseteq K$ set $I_{v}=\left(I R_{v}\right) \cap R$. Then the integral closure of $I$ is $\bar{I}=\bigcap_{v} I_{v}$, where the intersection ranges on all valuation rings in $K$ with center in $R$.

Proposition 3.2. Let $\varphi: X^{\prime} \rightarrow X$ be a proper birational morphism and let $\mathcal{I} \subseteq \mathcal{O}_{X}$ be a sheaf of ideals. Then $\overline{\mathcal{I}}=\left(\overline{\mathcal{I} \mathcal{O}_{X^{\prime}}}\right) \cap \mathcal{O}_{X}$.

Proof. It is a consequence of Lemma 3.1 and the valuative criterion of properness [9, Theorem 4.7, Section II].

Definition 3.3. A desingularization of $X$ is a proper birational morphism $\varphi$ : $X^{\prime} \rightarrow X$ such that

(i) $X^{\prime}$ is non-singular;

(ii) the morphism $\varphi$ is an isomorphism outside the singular locus of $X$. That is, if $U=X \backslash \operatorname{Sing}(X)$ and $U^{\prime}=\varphi^{-1}(U)$, then $U^{\prime} \cong U$ via $\varphi$.

Assume that $X \subseteq W$, where $W$ is a non-singular scheme. An embedded desingularization of $X \subseteq W$ is a proper birational morphism $\Pi: W^{\prime} \rightarrow W$ such that

(i) $W^{\prime}$ is non-singular;

(ii) the morphism $\Pi$ is an isomorphism outside the singular locus of $X$. That is, if $U=W \backslash \operatorname{Sing}(X)$ and $U^{\prime}=\Pi^{-1}(U)$, then $U^{\prime} \cong U$ via $\Pi$;

(iii) $W^{\prime} \backslash U^{\prime}$ is a simple divisor with normal crossings: $W^{\prime} \backslash U^{\prime}=H_{1} \cup \cdots \cup H_{r}$; (iv) if $X^{\prime} \subseteq W^{\prime}$ is the strict transform of $X$ in $W^{\prime}$ then $X^{\prime}$ is non-singular and has only normal crossings with the divisor $W^{\prime} \backslash U^{\prime}$.

Definition 3.4. Let $W$ be non-singular scheme. A log-resolution of an ideal $\mathcal{I} \subseteq \mathcal{O}_{W}$ is a proper birational morphism $\Pi: W^{\prime} \rightarrow W$ such that

(i) $W^{\prime}$ is non-singular,

(ii) $\Pi$ is an isomorphism outside the support of $\mathcal{I}$. If $U=W \backslash S u p p(\mathcal{I})$ and $U^{\prime}=\Pi^{-1}(U)$ then $U^{\prime} \cong U$ via $\Pi$.

(iii) $W^{\prime} \backslash U^{\prime}$ is a simple divisor with normal crossings: $W^{\prime} \backslash U^{\prime}=H_{1} \cup \cdots \cup H_{r}$.

(iv) The total transform of $\mathcal{I}$ in $W^{\prime}$ is a monomial with support in $W^{\prime} \backslash U^{\prime}$

$$
\mathcal{I} \mathcal{O}_{W^{\prime}}=\mathbf{I}\left(H_{1}\right)^{a_{1}} \cdots \mathbf{I}\left(H_{r}\right)^{a_{r}}
$$

Remark 3.5. It was proved by Hironaka in [10] that embedded desingularizations and log-resolutions do exist without restriction on the dimension of schemes over a field of characteristic zero. In fact, Hironaka proved that the 
morphism $\Pi$ may be obtained as a sequence of blowing-ups along regular centers.

The proof in [10] is existential. Constructive proofs may be found in [18] and also in [3]. If the characteristic of the ground field $k$ is positive, then resolution of singularities is an open problem for general dimension. The reader may found more details in [8]. We refer to [2] for constructive proofs of embedded desingularization of schemes, log-resolution of ideals and (non-embedded) desingularization of schemes.

Algorithms implementing resolution of singularities (in characteristic zero) in the computer are available for explicit computations. We will use the implementation of [4] available at http://www.risc.uni-linz.ac.at/projects/basic/ adjoints/blowup and implemented in Singular [7] and Maple. There is another implementation of resolution of singularities in [5] also implemented in Singular.

Proposition 3.6. Let us consider a log-resolution of $\mathcal{I} \subseteq \mathcal{O}_{W}$, as in Definition 3.4. Then

$$
\overline{\mathcal{I}^{m}}=\mathbf{I}\left(H_{1}\right)^{m a_{1}} \cdots \mathbf{I}\left(H_{r}\right)^{m a_{r}} \cap \mathcal{O}_{W},
$$

for any integer $m \geqslant 1$.

Proof. It is a consequence of Proposition 3.2 and the fact that locally principal ideals are integrally closed.

4. The reduced order of a sheaf and Łojasiewicz exponents. As in the previous section, here $X$ will denote an integral separated scheme of finite type over a field $k$.

Definition 4.1. Let $\mathcal{I}, \mathcal{J} \subseteq \mathcal{O}_{X}$ be two sheaves of ideals. We define two functions $\bar{\nu}_{\mathcal{I}}(\mathcal{J}): X \rightarrow \overline{\mathbb{R}}_{0}$ and $\mu_{\mathcal{I}}(\mathcal{J}): X \rightarrow \overline{\mathbb{R}}_{0}$ as follows

$$
\bar{\nu}_{\mathcal{I}}(\mathcal{J})(x)=\bar{\nu}_{\mathcal{I}_{x}}\left(\mathcal{J}_{x}\right)=\inf _{f \in \mathcal{J}_{x}} \bar{\nu}_{\mathcal{I}_{x}}(f), \quad \mu_{\mathcal{I}}(\mathcal{J})(x)=\mu_{\mathcal{I}_{x}}\left(\mathcal{J}_{x}\right)=\inf _{f \in \mathcal{J}_{x}} \mu_{\mathcal{I}_{x}}(f),
$$

for all $x \in X$.

We say that a function $\mu: X \rightarrow \mathbb{R} \cup\{\infty\}$ is lower-semicontinuous if for any $\alpha \in \mathbb{R}$, the set $F_{\alpha}=\{x \in X \mid \mu(x) \leqslant \alpha\}$ is closed. Analogously, we say that $\mu$ is upper-semicontinuous when the set $G_{\alpha}=\{x \in X \mid \mu(x) \geqslant \alpha\}$ is closed, for all $\alpha \in \mathbb{R}$.

Lemma 4.2. Assume that $X$ is non-singular and that $H_{1}, \ldots, H_{r}$ are nonsingular irreducible hypersurfaces having only normal crossings. Let $\lambda_{1}, \ldots, \lambda_{r}$ $\in \overline{\mathbb{R}}_{0}$ and let $\overline{\mathbb{N}}=\mathbb{N} \cup\{\infty\}$. Let us consider the function $\lambda_{i}: X \rightarrow \overline{\mathbb{N}}$ given by

$$
\lambda_{i}(x)= \begin{cases}\lambda_{i}, & \text { if } x \in H_{i}, \\ \infty, & \text { otherwise. }\end{cases}
$$

Then the function $\lambda: X \rightarrow \overline{\mathbb{N}}$ defined by $\lambda=\min \left\{\lambda_{i} \mid i=1, \ldots, N\right\}$ is lower-semicontinuous. 
Proof. Let $\alpha \in \overline{\mathbb{R}}_{0}$ and let us consider the set $F_{\alpha}=\{x \in X \mid \lambda(x) \leqslant \alpha\}$. We observe that $F_{\alpha}$ is the union of the hypersurfaces $H_{i}$ such that $\lambda_{i} \leqslant \alpha$. Therefore $F_{\alpha}$ is closed and the result follows.

Let $\mathcal{I}, \mathcal{J} \subseteq \mathcal{O}_{X}$ be two sheaves of ideals. Let $\Pi^{\prime}: X^{\prime \prime} \rightarrow X$ be a desingularization of $X$ (in the sense of Definition 3.3) and let $\Pi^{\prime \prime}: X^{\prime} \rightarrow X^{\prime \prime}$ be a log-resolution of $\mathcal{I} \mathcal{O}_{X^{\prime \prime}}$ (as in Definition 3.4), so that

$$
\mathcal{I} \mathcal{O}_{X^{\prime}}=\mathbf{I}\left(H_{1}\right)^{a_{1}} \cdots \mathbf{I}\left(H_{r}\right)^{a_{r}}
$$

for some positive integers $a_{1}, \ldots, a_{r}$. The total transform $\mathcal{J O}_{X^{\prime}}$ can be expressed as

$$
\mathcal{J} \mathcal{O}_{X^{\prime}}=\mathbf{I}\left(H_{1}\right)^{b_{1}} \cdots \mathbf{I}\left(H_{r}\right)^{b_{r}} \mathcal{J}^{\prime}
$$

where $\mathcal{J}^{\prime} \subseteq \mathcal{O}_{X^{\prime}}$ and $\mathcal{J}^{\prime} \nsubseteq \mathbf{I}\left(H_{i}\right)$, for all $i=1, \ldots, r$.

Proposition 4.3. In the setup described above, let us consider the function $\lambda=\min \left\{\frac{b_{i}}{a_{i}} \mid i=1, \ldots, r\right\}$. Then

$$
\mu_{\mathcal{I}}(\mathcal{J})(x)=\min \left\{\lambda\left(x^{\prime}\right) \mid x^{\prime} \in \Pi^{-1}(x)\right\},
$$

for all $x \in X$, and the function $\mu_{\mathcal{I}}(\mathcal{J})$ is lower-semicontinuous.

Proof. Let $p, q$ be positive integers. We observe that $\left(\mathcal{J}^{q}\right)_{x} \subseteq\left(\overline{\mathcal{I}^{p}}\right)_{x}$ if and only if $\left(\mathcal{J}^{q} \mathcal{O}_{X^{\prime}}\right)_{x^{\prime}} \subseteq\left(\mathcal{I}^{p} \mathcal{O}_{X^{\prime}}\right)_{x^{\prime}}$, for all $x^{\prime} \in \Pi^{-1}(x)$. Moreover, according to (4.1) and (4.2), we have the following equivalences:

$$
\begin{aligned}
\left(\mathcal{J}^{q} \mathcal{O}_{X^{\prime}}\right)_{x^{\prime}} \subseteq\left(\mathcal{I}^{p} \mathcal{O}_{X^{\prime}}\right)_{x^{\prime}} & \Longleftrightarrow\left(\mathbf{I}\left(H_{1}\right)^{q b_{1}} \cdots \mathbf{I}\left(H_{r}\right)^{q b_{r}} \mathcal{J}^{\prime q}\right)_{x^{\prime}} \\
& \subseteq\left(\mathbf{I}\left(H_{1}\right)^{p a_{1}} \cdots \mathbf{I}\left(H_{r}\right)^{p a_{r}}\right)_{x^{\prime}} \\
& \Longleftrightarrow\left(\frac{b_{i}}{a_{i}}\right)\left(x^{\prime}\right) \geqslant \frac{p}{q}, \quad i=1, \ldots, r \\
& \Longleftrightarrow \lambda\left(x^{\prime}\right) \geqslant \frac{p}{q} .
\end{aligned}
$$

Hence

$$
\mu_{\mathcal{I}}(\mathcal{J})(x) \geqslant \frac{p}{q} \Longleftrightarrow \lambda\left(x^{\prime}\right) \geqslant \frac{p}{q}, \quad \text { for all } x^{\prime} \in \Pi^{-1}(x),
$$

and we have $\mu_{\mathcal{I}}(\mathcal{J})(x)=\min \left\{\lambda\left(x^{\prime}\right) \mid x^{\prime} \in \Pi^{-1}(x)\right\}$.

The lower-semicontinuity of $\mu_{\mathcal{I}}(\mathcal{J})$ follows from the properness of $\Pi$.

As an immediate consequence of the previous theorem we obtain the following result.

Corollary 4.4. The value $\mu_{\mathcal{I}}(\mathcal{J})(x)$ is rational, for every $x \in X$.

Theorem 4.5. Let $\mathcal{I}, \mathcal{J} \subseteq \mathcal{O}_{X}$ be two sheaves of ideals. Then the functions $\bar{\nu}_{\mathcal{I}}(\mathcal{J})$ and $\mu_{\mathcal{I}}(\mathcal{J})$ are equal.

Proof. We use the same notation as in Proposition 4.3. Let us fix a point $x \in X$. First we prove that $\mu_{\mathcal{I}}(\mathcal{J}) \geqslant \bar{\nu}_{\mathcal{I}}(\mathcal{J})$.

Set $c_{n}=\nu_{\mathcal{I}}\left(\mathcal{J}^{n}\right)(x)$, for all $n \geqslant 1$. We observe that

$$
\bar{\nu}_{\mathcal{I}}(\mathcal{J})(x)=\sup _{n \in \mathbb{N}} \frac{c_{n}}{n} .
$$


By definition we have $\mathcal{J}^{n} \subseteq \mathcal{I}^{c_{n}} \subseteq \overline{\mathcal{I}^{c_{n}}}$, which implies that

$$
\mu_{\mathcal{I}}(\mathcal{J})(x) \geqslant \frac{c_{n}}{n}, \quad \text { for all } n \in \mathbb{N} \text {. }
$$

Therefore

$$
\mu_{\mathcal{I}}(\mathcal{J})(x) \geqslant \bar{\nu}_{\mathcal{I}}(\mathcal{J}) .
$$

Conversely, set $\frac{p}{q}=\mu_{\mathcal{I}}(\mathcal{J})(x)$. This implies that $\mathcal{J}_{x}^{q} \subseteq \overline{\mathcal{I}^{p}} x$. By Lemma 2.5 we have that $\bar{\nu}_{\mathcal{I}^{p}}\left(\mathcal{J}^{q}\right)(x) \geqslant 1$ and from Lemma 2.4 we obtain $\bar{\nu}_{\mathcal{I}}(\mathcal{J})(x) \geqslant \frac{p}{q}$.

Corollary 4.6. The value $\bar{\nu}_{\mathcal{I}}(\mathcal{J})(x)$ is rational, for every $x \in X$.

Definition 4.7. Let $X$ be a scheme as above with structure of complex variety. Let $\mathcal{I} \subseteq \mathcal{O}_{X}$ be a coherent sheaf of ideals and $K \subseteq X$ be a compact set. Let $f \in \Gamma\left(X, \mathcal{O}_{X}\right)$. The Eojasiewicz exponent of $f$ with respect to $\mathcal{I}$ at $K$, denoted by $\theta_{K}(f, \mathcal{I})$, is defined as the infimum of those $\theta \in \mathbb{R}_{+}$such that there exists an open set $U \subseteq \mathbb{C}^{n}$ such that $K \subseteq U$ and a constant $C \geqslant 0$ such that

$$
|f(x)|^{\theta} \leqslant C \cdot \sup _{g \in \Gamma(U, \mathcal{I})}|g(x)|,
$$

for all $x \in U$.

If $\mathcal{J} \subseteq \mathcal{O}_{X}$ is a sheaf of ideals, then

$$
\theta_{K}(\mathcal{J}, \mathcal{I})=\sup _{f \in \Gamma(X, \mathcal{J})} \theta_{K}(f, \mathcal{I}) .
$$

Theorem 4.8. $[14,6.3]$ Under the hypothesis of the previous definition we have

$$
\theta_{K}(\mathcal{J}, \mathcal{I})=\frac{1}{\bar{\nu}_{\mathcal{I}}(\mathcal{J})(K)},
$$

where $\bar{\nu}_{\mathcal{I}}(\mathcal{J})(K)=\min \left\{\bar{\nu}_{\mathcal{I}}(\mathcal{J})(x) \mid x \in K\right\}$.

As a direct consequence of the previous theorem and of Corollary 4.6 we obtain that the Łojasiewicz exponent $\theta_{K}(\mathcal{J}, \mathcal{I})$ is a rational number.

Definition 4.9. Let $\mathcal{I}, \mathcal{J} \subseteq \mathcal{O}_{X}$ be two sheaves of ideals. We define the function $\theta(\mathcal{J}, \mathcal{I}): X \rightarrow \mathbb{Q}$ as follows:

$$
\theta(\mathcal{J}, \mathcal{I})(x)=\theta_{\{x\}}(\mathcal{J}, \mathcal{I}),
$$

for all $x \in X$.

From Proposition 4.3 and Theorem 4.8 we obtain that the function $\theta(\mathcal{J}, \mathcal{I})$ : $X \rightarrow \mathbb{Q}$ is upper-semicontinuous.

5. Computation of Łojasiewicz exponents for isolated singularities. Let $W$ be an scheme with structure a regular analytic variety. Let $\mathcal{I}$ be a sheaf of ideals in $\mathcal{O}_{W}$ such that $\operatorname{Supp}(\mathcal{I})=\{x\}$, where $x \in W$. We define the Lojasiewicz exponent of $\mathcal{I}$ at $x$ as $\mathcal{L}_{x}(\mathcal{I})=\theta(\mathcal{J}, \mathcal{I})(x)$ where $\mathcal{J}$ is the sheaf of ideals

$$
\mathcal{J}_{y}= \begin{cases}\mathfrak{m}_{x} & \text { if } y=x \\ 1 & \text { if } y \neq x .\end{cases}
$$

Theorem 5.1. The Eojasiewicz exponent of $\mathcal{I}$ is determined by the total transform of $\mathfrak{m}_{x}$ via the log-resolution of $\mathcal{I}$. 
Proof. Let us consider a log-resolution of $\mathcal{I}$ as in Definition 3.4. The morphism $W^{\prime} \rightarrow W$ is a sequence of blowing-ups along regular centers:

$$
W=W_{0} \longleftarrow W_{1} \longleftarrow \cdots \longleftarrow W_{r}=W^{\prime} .
$$

We observe that the first blowing-up must have $\operatorname{Supp}(\mathcal{I})$ as center. Therefore $\mathcal{J} \mathcal{O}_{W_{1}}=\mathfrak{m}_{x} \mathcal{O}_{W_{1}}=I\left(H_{1}\right)$ and the total transform of $\mathfrak{m}_{x}$ is a monomial, that is

$$
\mathfrak{m}_{x} \mathcal{O}_{W^{\prime}}=\mathbf{I}\left(H_{1}\right)^{b_{1}} \cdots \mathbf{I}\left(H_{r}\right)^{b_{r}},
$$

for some positive integers $b_{1}, \ldots, b_{r}$.

Let us suppose that the total transform of $\mathcal{I}$ in $W^{\prime}$ is written as in (3.1). Then, we obtain the following equivalences:

$$
\begin{aligned}
\mathfrak{m}_{x}^{p} \subseteq \overline{\mathcal{I}^{q}} & \Longleftrightarrow \mathbf{I}\left(H_{1}\right)^{p b_{1}} \cdots \mathbf{I}\left(H_{r}\right)^{p b_{r}} \subseteq \mathbf{I}\left(H_{1}\right)^{q a_{1}} \cdots \mathbf{I}\left(H_{r}\right)^{q a_{r}} \\
& \Longleftrightarrow p b_{i} \geqslant q a_{i}, \quad i=1, \ldots, r \\
& \Longleftrightarrow \frac{p}{q} \geqslant \frac{a_{i}}{b_{i}}, \quad i=1, \ldots, r .
\end{aligned}
$$

Then, we conclude that

$$
\mathcal{L}_{x}(\mathcal{I})=\max \left\{\frac{a_{i}}{b_{i}}, i=1, \ldots, r\right\} .
$$

By $(5.1)$, the problem of computing $\mathcal{L}_{x}(\mathcal{I})$ reduces to determine the integers $a_{i}, b_{i}$, for $i=1, \ldots, r$, which in turn, come from determining the total transform of $\mathfrak{m}_{x}$ via the log-resolution of $\mathcal{I}$. Next we expose some examples in the ring $\mathcal{O}_{n}$ of holomorphic gems $f:\left(\mathbb{C}^{n}, 0\right) \rightarrow \mathbb{C}$.

Example 1. Let us consider the ideal $I$ of $\mathcal{O}_{3}$ generated by the polynomials

$$
\begin{aligned}
& g_{1}=x^{4}+x y z+y^{4} \\
& g_{2}=x y^{2} z \\
& g_{3}=y^{5}+z^{5} .
\end{aligned}
$$

Then, applying relation $(5.1)$, it follows that $\mathcal{L}_{0}(I)=5+\frac{5}{6}$. Let us denote by $e(I)$ the Samuel multiplicity of $I$. The same value for $\mathcal{L}_{0}(I)$ is obtained by following the approach explained in Section 4 of [1], since $e(I)$ equals the Rees mixed multiplicity of the ideals $I_{1}=\left\langle x^{4}, x y z, y^{4}\right\rangle, I_{2}=\left\langle x y^{2} z\right\rangle$ and $I_{3}=\left\langle y^{5}, z^{5}\right\rangle$, which is equal to 80 .

Example 2. Let us consider the function $f \in \mathcal{O}_{3}$ given by $f(x, y, z)=y^{6}+z^{4}+$ $x(x-3 z)^{2}$ and let us denote by $\mu(f)$ the Milnor number of $f$. We observe that $f$ is a Newton degenerate function in the sense of [12]. Moreover $\mu(f)=25$, whereas the Newton number of the Newton polyhedron of $f$ is equal to 20 . Therefore, the Eojasiewicz exponent of $f$ can not be computed using the technique explained in [1] via mixed multiplicities of monomial ideals.

Using relation (5.1) we obtain

$$
\mathcal{L}_{0}(\nabla f)=5 .
$$


Therefore, by virtue of [17], the degree of topological determinacy of $f$ is given by

$$
\left[\mathcal{L}_{0}(\nabla f)\right]+1=6 .
$$

The examples above have been computed with the program avalaible at http://www.math.arq.uva.es/sencinas/ingles.html

\section{References}

[1] C. Bivià-Ausina, Local Łojasiewicz exponents, Milnor numbers and mixed multiplicities of ideals, Math. Z. 262 (2009), 389-409.

[2] A. M. Bravo, S. Encinas, and O. Villamayor, A simplified proof of desingularization and applications, Rev. Mat. Iberoamericana 21 (2005), 349-458.

[3] E. Bierstone and P. D. Milman, A simple constructive proof of canonical resolution of singularities, Effective Methods in Algebraic Geometry (Castiglioncello, 1990), Progr. Math. 94, Birkhäuser Boston, Boston, MA, 1991, pp. 11-30.

[4] G. Bodnár And J. Schicho, Automated resolution of singularities for hypersurfaces, J. Symbolic Comput. 30 (2000), 401-428.

[5] A. Frühbis-Krüger and G. Pfister, Algorithmic resolution of singularities, in: Singularities and computer algebra, London Math. Soc. Lecture Note Ser., 324, pp. 157-183. Cambridge University Press, Cambridge, 2006.

[6] T. Fukui, Łojasiewicz type inequalities and Newton diagrams, Proc. Amer. Math. Soc. 112 (1991), 1169-1183.

[7] G.-M. Greuel, G. Pfister, and H. Schönemann, Singular 3.0, A Computer Algebra System for Polynomial Computations, Centre for Computer Algebra, University of Kaiserslautern, 2005, http://www.singular.uni-kl.de.

[8] H. Hauser, The Hironaka theorem on resolution of singularities (or: A proof we always wanted to understand), Bull. Amer. Math. Soc. (N.S.) 40 (2003), 323-403.

[9] R. Hartshorne, Algebraic Geometry, Graduated Texts in Math. 52 (Springer), 1983

[10] H. Hironaka, Resolution of singularities of an algebraic variety over a field of characteristic zero. I, II, Ann. Math. (2) 79 (1964), 109-203; 79 (1964), 205-326.

[11] C. Huneke and I. Swanson, Integral Closure of Ideals, Rings, and Modules, London Mathematical Society Lecture Note Series, 336, Cambridge University Press, Cambridge, 2006.

[12] A. G. Kouchnirenko, Polyèdres de Newton et nombres de Milnor, Invent. Math. 32 (1976), 1-31.

[13] A. Lenarcik, On the Łojasiewicz exponent of the gradient of a holomorphic function, Singularities Symposium-Lojasiewicz 70 (Kraków, 1996; Warsaw, 1996), Banach Center Publ., 44, pp. 149-166, Polish Acad. Sci., Warsaw, 1998.

[14] M. Lejeune And B. Teissier, Clôture intégrale des idéaux et équisingularité, Centre de Mathématiques, Université Scientifique et Medicale De Grenoble (1974). arXiv:0803.2369v1[math.CV] 
[15] A. PŁoski, Multiplicity and the Łojasiewicz exponent, Singularities (Warsaw, 1985), Banach Center Publ., 20, pp. 353-364, Pwn, Warsaw, 1988.

[16] D. ReEs, Valuations associated with a local ring II, J. London Math. Soc. 31 (1956), 228-235.

[17] B. Teissier, Variétés polaires I. Invariants polaires des singularités d'hypersurfaces, Invent. Math. 40 (1977), 267-292.

[18] O. Villamayor, Constructiveness of Hironaka's resolution. Ann. Sci. École Norm. Sup. (4) 22 (1989), 1-32.

C. Bivì̀-Ausina

Institut Universitari de Matemàtica Pura i Aplicada, Universitat Politècnica de València,

Camí de Vera,

s/n 46022 Valencia, Spain

e-mail: carbivia@mat.upv.es

\section{S. ENCINAS}

Departamento de Matemática Aplicada, Universidad de Valladolid, Avda. Salamanca, s/n 47014 Valladolid, Spain.

e-mail: sencinas@maf .uva.es

Received: 11 March 2009 